\title{
Eigen Decomposition of Reed Muller Transform Using Kronecker Method
}

\author{
Jyotsna Singh \\ Division of Electronics and Comm. Engg. \\ Netaji Subhas Institute of Technology \\ Sector 3, Dwarka \\ New Delhi 110075, India
}

\author{
Shikha Garg \\ Division of Electronics and Comm. Engg. \\ Netaji Subhas Institute of Technology \\ Sector 3, Dwarka \\ New Delhi 110075, India
}

\begin{abstract}
Spectral methods have been applied to many areas of digital system design. Reed-Muller Transform (RMT) is a spectral transform which is self inverse in nature. In this paper, eigendecomposition of Reed-Muller Transform using Kronecker Product method is introduced. The properties of eigenvectors and eigenvalues of RMT are also illustrated.
\end{abstract}

\section{Keywords}

eigenvalue, eigenvector, Kronecker Product, Reed Muller Transform.

\section{INTRODUCTION}

Many spectral transforms have been extensively studied in literature such as Walsh, Reed-Muller, Arithmetic, Haar transforms. The Reed-Muller Transform (RMT) has been motivated by the work of Reed and Muller [1], [2]. Reed-Muller is an important spectral transform that has found its application in areas like fault detection, testing [3] and image processing [4]. This led to considerable interest in the AND-XOR expansion of Boolean functions [5], [6]. Since Reed-Muller transform is so widely used, it will be useful to find algorithms to perform its eigen-decomposition. Eigenfunctions of Reed-Muller Transform were introduced by Sasao and Butler in [7]. The work identified three symmetric functions with certain special properties and showed that these symmetric functions can be found among the eigenfunctions of the Reed-Muller transform. The eigenvectors of the Reed-Muller Transform were evaluated using canonical sum of products. Tseng [8] proposed eigen decomposition of Hadamard transform using kronecker product method. Other orthogonal transforms can also be decomposed using similar technique. In this paper, we propose a method to find the eigenvectors and eigenvalues of Reed-Muller transform using the Kronecker Product method. Also, the properties of kronecker product, eigenvalues and eigenvectors are discussed and illustrated for RMT.

The paper is organized as: In Section-II, Kronecker product and its properties are discussed. Section-III describes the ReedMuller expansion and its properties. Reed-Muller transform is also defined in same section. The eigen decomposition of RMT using Kronecker product method is described in section-IV. An example is illustrated for RMT with three variables.

\section{KRONECKER PRODUCT}

Kronecker product representations lead to efficient implementations of numerous discrete orthogonal transforms. The kronecker products can be defined in terms of matrix factorizations [9].

If $\mathbf{A}$ is an $m \times m$ matrix and $\mathbf{B}$ is a $n \times n$ matrix, then the Kronecker product (or Tensor product) of $\mathbf{A}$ and $\mathbf{B}$ is $\boldsymbol{A} \otimes \boldsymbol{B}_{\text {which is an }} m \times n m$ matrix given by

$\boldsymbol{A} \otimes \boldsymbol{B}=\left[\begin{array}{ccc}a_{11} \boldsymbol{B} & \ldots & a_{1 \mathrm{~m}} \boldsymbol{B} \\ \vdots & \cdots & \vdots \\ a_{\mathrm{m} 1} \boldsymbol{B} & \cdots & a_{\mathrm{mm}} \boldsymbol{B}\end{array}\right]$

here $a_{i j}$ are the elements of matrix $\boldsymbol{A}$ of row $i$ and column $j$.

Kronecker product satisfies the following properties:

Property 1: For all $T \in R^{n \times n}$ and $S \in R^{m \times m}$, $(T \otimes S)^{T}=T^{T} \otimes S^{T}$, here $(.)^{T}$ represents transpose of the matrix.

Property 2: If $\mathbf{T}$ and $\mathbf{S}$ are real symmetric matrices of size $n \times n$ and $m \times m$ then $\boldsymbol{T} \otimes \boldsymbol{S}$

is also a symmetric matrix of size $n m \times n m$

Property 3: If $\mathbf{T}$ and $\mathbf{S}$ are real orthogonal matrices then $\boldsymbol{T} \otimes \boldsymbol{S}_{\text {will also be an orthogonal matrix. }}$

Property 4: Let $\mathbf{T}$ be a matrix of size $n \times n$ with eigenvectors $\left(t_{1}, \ldots, t_{n}\right)$ and eigenvalues $\left(\lambda_{1}, \ldots, \lambda_{n}\right)$. Another matrix $\mathbf{S}$ of size $m \times m$ has eigenvectors $\left(s_{1, \ldots,} s_{m}\right)$ and eigenvalues $\left(\mu_{1}, \ldots, \mu_{m}\right)$. Then $\lambda_{i} \mu_{j}$ and $\boldsymbol{t}_{i} \otimes \boldsymbol{s}_{j}$ will be eigenvalues and eigenvectors of matrix $\boldsymbol{T} \otimes \boldsymbol{S}$ respectively, where $i=1,2, \ldots, n$ and $j=1,2, \ldots, m$.

\section{REED-MULLER TRANSFORM}

\section{A. Reed-Muller expansion}

An expression which can represent any boolean function as a modulo-2 sum of products, is known as the compliment-free ring-sum or Reed-Muller expression (RME). Reed-Muller expansions are advantageous for many important boolean functions in terms of area, speed and testability point of view [10]. A function $\mathrm{f}($.$) with n$ variables can be defined by $2^{n}$ terms in a sum of products form as in equation 


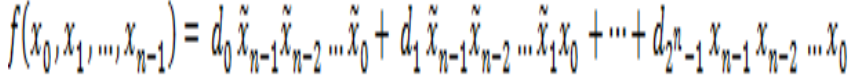

here $\left(d_{0}, d_{1}, \ldots, d_{2} n-1\right)$ represents the values in the output column of the truth table of the function. These coefficients can be represented in vector form $\mathbf{D}$, called truth vector. Using the positive davio expansion [10], as given in equation $f\left(x_{0}, x_{1}, \ldots, x_{n-1}\right)=f_{0} \oplus x_{0} f_{2}$

Here $\oplus$ denotes modulo-2 addition. $f_{0}=f\left(0, x_{1, \ldots,} x_{n-1}\right), f_{1}=f\left(1, x_{1}, \ldots, x_{n-1}\right)$ and $f_{2}=f_{0} \oplus f_{1}$.

The Reed-Muller expansion can be written as $f\left(x_{0}, x_{1}, \ldots, x_{n-1}\right)=a_{0} \oplus a_{1} x_{0} \oplus \ldots \oplus a_{1,2, n} x_{0} x_{1} \ldots x_{n-1}$

This is positive polarity Reed-Muller expansion (PPRM). The negative davio expansion, is given as

$f\left(x_{0}, x_{1}, \ldots, x_{n-1}\right)=\tilde{x}_{0} f_{2} \oplus f_{1}$

Using (5), the Reed Muller expansion for the function can be obtained

$$
f\left(x_{0}, x_{1}, \ldots, x_{n-1}\right)=a_{0} \oplus a_{1} \tilde{x}_{0} \oplus \ldots \oplus a_{1,2, n n} \tilde{x}_{0} \tilde{x}_{1} \ldots \tilde{x}_{n-1}
$$

This is negative polarity Reed-Muller Expansion (NPRM). From (4) we observe that in PPRM

all the variables are in positive form. The expression can be converted to NPRM by substituting

variable $x_{\tilde{i}}$ with its negation $\tilde{\boldsymbol{x}}_{\bar{i}}$ as shown in (6). In this way the canonical form of the Reed-

Muller expansion is retained. If each variable is restricted to retain the same polarity in all the terms of the expansion, that is, either positive or negative but not both, the canonical form is called the Fixed Polarity Reed Muller (FPRM) form. For a function with $n$ variables, the number of possible arrangements of polarities is $2^{n}$.

\section{B. Reed-Muller Transform}

A Reed-Muller Transformation matrix of $n$ variables $\mathbf{R}(\mathrm{n})$ can be recursively written as

$$
\mathbf{R}(n)=\left[\begin{array}{lc}
\boldsymbol{R}(n-1) & 0 \\
\boldsymbol{R}(n-1) & \boldsymbol{R}(n-1)
\end{array}\right] \text { for } n \geq 1
$$

and $R(0)=1$. Thus, the Reed-Muller transformation matrix of one variable, $\mathbf{R}(1)$ is

$\mathbf{R}(1)=\left[\begin{array}{ll}1 & 0 \\ 1 & 1\end{array}\right]$ (8)

In this case the matrix multiplication is over the field GF(2). The Reed-Muller transform matrix can be recursively defined using kronecker product [11], [12] as

$$
\begin{aligned}
R(n) & =R(1) \otimes R(1) \otimes \ldots \otimes R(1) \\
& =\otimes^{n} R(1)
\end{aligned}
$$

The eigen decomposition of the matrix $\mathbf{R}(1)$ is given as

$$
R(1)=V(1) D(1) V(1)^{-1}
$$

where $V(1)=\left[\begin{array}{cc}0 & 0 \\ 1 & -1\end{array}\right]$ is the eigenvector matrix for $\mathbf{R}(1)$. The columns of matrix represent eigenvectors $\boldsymbol{v}_{10}$ and $\boldsymbol{v}_{11}$. Similarly matrix $D(1)=\left[\begin{array}{ll}1 & 0 \\ 0 & 1\end{array}\right]$ is eigenvalue matrix for $\mathbf{R}(1)$. The columns of eigenvalue matrix are named as $\lambda_{10}$ and $\lambda_{11}$.

\section{EIGEN VECTOR DECOMPOSITION OF REED-MULLER TRANSFORM (RMT)}

We propose a method to compute the eigenvectors and eigenvalues of the RMT matrix, $\mathbf{R}(n)$, by using the Kronecker product method. The eigenvector $\mathbf{x}$ for any matrix $\mathbf{M}$ is calculated using the characteristic equation $M x=\lambda x$, where $\lambda$ is the eigenvalue for matrix $\mathbf{M}$. The eigenvectors and eigenvalues of RMT follow the definition given below, as mentioned in [7].

Definition 4.1: Let $\mathbf{v}$ be a binary vector of $2^{n}$ elements and $\mathbf{R}(n)$ be the Reed-Muller Transformation matrix of $\mathrm{n}$ variables. Then, a vector $\mathbf{v}$ satisfying $\boldsymbol{R}(n) \boldsymbol{v}=\boldsymbol{v}$ is an eigenvector of ReedMuller transform. Eigenvalue $\lambda$ exhibits only 1 as its nonzero value for RMT.

The RMT matrix $\mathbf{R}(\mathrm{n})$ can be decomposed using the kronecker product as given by (9). The matrix $\mathbf{R}(n)$ has $2^{n}$ eigenvectors which can be computed using the kronecker product method. Let an index $p=0,1, \ldots, 2^{n}-1$ whose binary representation is given as $d_{n-1} d_{n-2} \ldots d_{1} d_{0}$ where $d_{i}$ are the binary bits 0 and 1 . We can show that Reed-Muller Transform exhibits following properties:

- $\quad$ Property 1: The eigenvectors of RMT matrix can be obtained using the equation

$$
v_{n p}=v_{1 d_{n-1}} \otimes v_{1 d_{n-2}} \otimes \ldots v_{1 d_{1}} \otimes v_{1 d_{0}}
$$


using the above expansion it can be shown that the eigenvectors of RMT are always a combination of 0 and 1 or 0 and -1 .

- $\quad$ Property 2: The matrix $\mathbf{R}(\mathrm{n})$ has $2^{n}$ eigenvalues whose column matrix can be obtained as

$\lambda_{n, i}=\lambda_{1 d_{n-1}} \otimes \lambda_{1 d_{n-2}} \otimes \ldots \otimes \lambda_{1 d_{1}} \otimes \lambda_{1 d_{0}}$

for $0 \leq i \leq 2^{n}-1$. Using (12) it can be shown that the eigenvalues of RMT matrix are always 1 .

- $\quad$ Property 3: The norm of eigenvector $\boldsymbol{v}_{n, p}$ is given as $\left\|v_{n, p}\right\|=1 \quad$ for $p=0,1, \ldots, 2^{n}-1$ $-(13)$

Thus the eigenvectors for RMT matrix are orthonormal.

As an example, let us consider the Reed-Muller transform matrix $\mathbf{R}(3)$ for $n=3$. The desired transform can be obtained from kronecker product of $\mathbf{R}(1)$ i.e. $\boldsymbol{R}(3)=\boldsymbol{R}(1) \otimes \boldsymbol{R}(1) \otimes \boldsymbol{R}(1)$. By doing this we get

$\mathbf{R}(3)=\left[\begin{array}{llllllll}1 & 0 & 0 & 0 & 0 & 0 & 0 & 0 \\ 1 & 1 & 0 & 0 & 0 & 0 & 0 & 0 \\ 1 & 0 & 1 & 0 & 0 & 0 & 0 & 0 \\ 1 & 1 & 1 & 1 & 0 & 0 & 0 & 0 \\ 1 & 0 & 0 & 0 & 1 & 0 & 0 & 0 \\ 1 & 1 & 0 & 0 & 1 & 1 & 0 & 0 \\ 1 & 0 & 1 & 0 & 1 & 0 & 1 & 0 \\ 1 & 1 & 1 & 1 & 1 & 1 & 1 & 1\end{array}\right]$

Table-I shows the eigenvectors of R(3), computed using (11). These eigenvectors satisfy equation $\boldsymbol{R}(n) \boldsymbol{v}=\boldsymbol{v}$, as given by definition 4.1. Also the eigenvalues, are evaluated using (12). The values of $\lambda$ are found to be 1 as stated in property 2 (section IV)

\section{CONCLUSION}

In this paper, the eigenvector decomposition of Reed-Muller transform is investigated. A kronecker product method for finding eigenvalues and eigenvectors of Reed-Muller transform is described. Also, the properties of kronecker product, eigenvalues and eigenvectors are discussed for the given transform.

\begin{tabular}{|c|c|c|cccccc|}
\hline$v$ & $\begin{array}{c}\text { Kronecker } \\
\text { Product }\end{array}$ & \multicolumn{7}{|c|}{ Eigenvectors of $\boldsymbol{R}(3)$} \\
\hline$v_{30}$ & $v_{10} \otimes v_{10} \otimes v$ & {$[0$} & 0 & 0 & 0 & 0 & 0 & 0 \\
\hline$v_{31}$ & $v_{10} \otimes v_{10} \otimes v$ & {$[0$} & 0 & 0 & 0 & 0 & 0 & 0 \\
\hline
\end{tabular}

\begin{tabular}{|l|l|lllllll|}
\hline$v_{32}$ & $v_{10} \otimes v_{11} \otimes v$ & {$[0$} & 0 & 0 & 0 & 0 & 0 & 0 \\
\hline$v_{33}$ & $v_{10} \otimes v_{11} \otimes v$ & {$[0$} & 0 & 0 & 0 & 0 & 0 & 0 \\
\hline$v_{34}$ & $v_{11} \otimes v_{10} \otimes v$ & {$[0$} & 0 & 0 & 0 & 0 & 0 & 0 \\
\hline$v_{35}$ & $v_{11} \otimes v_{10} \otimes v$ & {$[0$} & 0 & 0 & 0 & 0 & 0 & 0 \\
\hline$v_{36}$ & $v_{11} \otimes v_{11} \otimes v$ & {$[0$} & 0 & 0 & 0 & 0 & 0 & 0 \\
\hline$v_{37}$ & $v_{11} \otimes v_{11} \otimes v$ & {$[0$} & 0 & 0 & 0 & 0 & 0 & 0 \\
\hline
\end{tabular}

\section{TABLE 1 KRONECKER PRODUCT OF EIGENVECTOR v OF R(3) WITH SIZE $8 \times 8$}

\section{REFERENCES}

[1] I. S. Reed, " A Class of Multiple-Error-Correcting Codes and the Decoding Scheme,"

IEEE Trans. Inf. Theory, vol. IT-4, pp. 38-49, 1954.

[2] D.E. Muller, “Application of Boolean Algebra to Switching Circuit Design and to Error

Detection," IRE Trans. Electronic Computers, vol. 3, pp. 6-12, 1954.

[3] T. Damarla and M. G. Karpovsky, " Reed-Muller spectral techniques for fault detection,"

IEEE Trans. Comput., vol. 38, no. 6, pp. 788-797, June 1989.

[4] B. J. Falkowski and B. T. Olejnicka, “ Multiple-valued and spectral approach to lossless

compression of binary, gray scale and color biomedical images," in Proc. 32nd IEEE Int. Symp.

Multiple- Valued Logic, Boston, Massachusetts, May 2002, pp.136-142.

[5] T. Sasao, "Logic Synthesis with EXOR Logic Gates", Logic Synthesis and optimization,

ed. Kluwer Academic, 1993.

[6] Sasao, T., "Logic Synthesis and Optimization," Ed., Boston: Kluwer, 1993.

[7] T. Sasao and J.T. Butler, "The eigenfunction of the ReedMuller transformation," RM-2007,

Oslo, Norway, May 16, 2007.

[8] C. C. Tseng., " Eigenvector and Fractionalization of Discrete Hadamard Transform," In Proceedings of IEEE International Symposium on Circuits and Systems, New Orleans, LA, May 2007, pp.2307-2310.

[9] Graham, A., "Kronecker products and matrix calculus with applications," Ellis Horwood

Limited and John Wiley and Sons, 1981.

[10] C.H. Chang and B. J. Falkowski, “ Flexible optimization of fixed polarity Reed-Muller

expansions for multiple output incompletely specified Boolean functions," Proc. Asia South Pacific Design Automation Conference, Makuhari, Japan, pp.335-340, Aug 1995.

[11] Kamran Iravani, Marek A. Perkowski, “ Image Compression based on Reed Muller

Transorm" in Proc. Int. Conf. on Computational Intelligence and Multimedia Applications, 1998, 
pp. 81-95.

[12] Whitney J. Townsend, Mitchell A Thornton, Rolf Drechsler, D. Michael Miller, " ComputingWalsh, Arithmetic, and Reed-Muller Spectral Decision Diagrams using Graph Transformations", GLSVLSI'02, April 18-19, 2002, New York, USA. 